Pacific Journal of Mathematics

SERIES EXPANSIONS OF ANALYTIC FUNCTIONS. II 


\section{SERIES EXPANSIONS OF ANALYTIC FUNCTIONS II}

\section{J. D. Buckholtz and J. K. Shaw}

This paper is concerned with series expansions of the form $f(z)=\sum_{0}^{\infty} h_{k} p_{k}(z)$, where the functions $\left\{p_{k}\right\}$ are analytic and satisfy a certain asymptotic condition. Relationships between the space $\mathscr{F}$ of expandable functions, the coefficient space $\mathscr{H}$, and the matrix operator $B_{j k}=p_{k}^{(j)}(0)$ are studied, and $\mathscr{F}$ is shown to be a Banach space isomorphic to $c_{0}$, the space of complex sequences with limit 0 . Necessary and sufficient conditions for convergence of $\Sigma_{0}^{\infty} h_{k} p_{k}(z)$ are given in terms of the coefficient sequence $h$.

Introduction. Let $\left\{p_{k}\right\}_{0}^{\infty}$ be a sequence of functions analytic on a region $\Omega$ which contains the point 0 . A function $f$ is said to possess a $\left\{p_{k}\right\}$ expansion if there exists a complex sequence $h=\left\{h_{k}\right\}_{0}^{\infty}$ such that

$$
\sum_{k=0}^{\infty} h_{k} p_{k}(z)
$$

converges uniformly to $f(z)$ on compact subsets of $\Omega$. The set of all such $f$ is the expansion class of $\left\{p_{k}\right\}$; we shall denote this set by $\mathscr{F}$, and denote by $\mathscr{H}$ the set of all sequences $h$ such that (1.1) is uniformly convergent on compact subsets of $\Omega$.

Let $Y$ denote the set of all complex sequences $y=\left\{y_{j}\right\}_{0}^{\infty}$ such that

$$
y_{\jmath}=f^{(j)}(0) \text {, }
$$$$
j=0,1,2, \cdots,
$$

for some $f \in \mathscr{F}$, and define the infinite matrix $B$ by

$$
B_{j k}=p_{k}^{(j)}(0), 0 \leqq j, k<\infty .
$$

We make no distinction between $B$ and the linear sequence-to-sequence operator it defines. It is the relationship between the operator $B$, the expansion class $\mathscr{F}$ and the coefficient space $\mathscr{H}$ that we study.

Suppose that $f \in \mathscr{F}$ is given by (1.1) and let $y=\left\{f^{(j)}(0)\right\}_{0}^{\infty}$. If we differentiate (1.1) $j$ times and set $z=0$ we obtain

$$
\begin{aligned}
y_{J}=f^{(j)}(0) & =\sum_{k=0}^{\infty} h_{k} p_{k}^{(j)}(0) \\
& =\sum_{k=0}^{\infty} B_{j k} h_{k}=(B h)_{j} .
\end{aligned}
$$


Therefore $y=B h$. It follows that the coefficient space $\mathscr{H}$ is contained in the domain of $B$ and the space $Y$ is contained in the range of $B$. In [2], the conditions imposed on $\left\{p_{k}\right\}_{0}^{\infty}$, which led to an asymptotic formula of the type

$$
p_{k} \approx \alpha_{k} \varphi
$$

where $\varphi$ is analytic in $\Omega$ and $\left\{\alpha_{k}\right\}_{0}^{\infty}$ is a complex sequence, were sufficiently strong to insure that the domain and range of $B$ were precisely $\mathscr{H}$ and $\mathscr{Y}$. Additionally, the expansion class $\mathscr{F}$ was shown to be a Banach space isomorphic to $c_{0}$, the space of complex sequences with limit 0 , normed with the supremum norm. In the present paper we relax certain of these conditions considerably and obtain the same results under assumptions leading to an asymptotic formula of the type

$$
p_{k} \approx \alpha_{k 1} \varphi_{1}+\alpha_{k 2} \varphi_{2}+\cdots+\alpha_{k m} \varphi_{m}
$$

where the functions $\varphi_{1}, \cdots, \varphi_{m}$ are analytic and linearly independent in $\Omega$. Typical examples exhibiting this behavior are found in the Bernoulli and Euler polynomials ([1]). For the Bernoulli polynomials one has

$$
p_{n}(z)=-\frac{e^{2 \pi i z}+(-1)^{n} e^{-2 \pi i z}}{(2 \pi i)^{n}}+O\left(\frac{e^{3 \pi|z|}}{(3 \pi)^{n-1}}\right), n \rightarrow \infty .
$$

In a number of classical polynomial expansions it happens that convergence at a single point, or on certain finite sets, implies uniform convergence on every compact set. This is the case with Lidstone polynomials [4], the Abel interpolation series [2], certain Appell polynomials [3], and the more general polynomials considered by W. T. Martin [5]. We show that the assumptions we place on $\left\{p_{k}\right\}_{0}^{\infty}$ imply a general result in this direction which includes most of those obtained previously as special cases.

2. Notation and statement of results. Let $d$ be a fixed positive integer and let $P_{k}$ denote the row matrix

$$
P_{k}(z)=\left[p_{k d}(z), p_{k d+1}(z), \cdots, p_{k d+d-1}(z)\right], k=0,1,2, \cdots, z \in \Omega \text {. }
$$

Our principal requirement on the sequence $\left\{p_{k}\right\}_{0}^{\infty}$ is that there exist a vector function

$$
\Phi(z)=\left[\varphi_{1}(z), \varphi_{2}(z), \cdots, \varphi_{d}(z)\right]
$$

and a sequence $\left\{\alpha_{k}\right\}_{0}^{\infty}$ of $d \times d$ invertible matrices such that 


$$
\lim _{k \rightarrow \infty} p_{k}(z) \alpha_{k}^{-1}=\Phi(z)
$$

for all $z \in \Omega$. We norm the space of $d$-dimensional row matrices with the norm

$$
\|R\|_{\rho}=\max _{1 \leq t \leq d}\left|R_{t}\right|
$$

the space of $d \times d$ matrices with the norm

$$
\|M\|_{G}=\sum_{i=1}^{d} \sum_{j=1}^{d}\left|M_{i j}\right|
$$

and the space of $d$-dimensional column vectors with the norm

$$
\|C\|_{\tau}=\max _{1 \leqq t \leqq d}\left|C_{t}\right|
$$

We require that

$$
\begin{gathered}
\sum_{k=0}^{\infty}\left\|P_{k}(z) \alpha_{k}^{-1}-P_{k-1}(z) \alpha_{k-1}^{-1}\right\|_{\rho} \text { converges } \\
\text { uniformly on compact subsets of } \Omega
\end{gathered}
$$

(we define $P_{-1}=0$ ) and we shall also need the requirement that the matrices $\left\{\alpha_{k}\right\}_{0}^{\infty}$ satisfy the "uniform invertibility" condition

$$
\sup _{0 \leqq k<\infty}\left\|\alpha_{k}\right\|_{G}\left\|\alpha_{k}^{-1}\right\|_{G}<\infty
$$

Condition (2.2) implies that the convergence in (2.1) is at least uniform on compact subsets of $\Omega$. Our last requirement is that

$$
\text { the functions } \varphi_{1}, \varphi_{2}, \cdots, \varphi_{d} \text { are }
$$

linearly independent.

We define a uniqueness set for functions $f_{1}, f_{2}, \cdots, f_{m}$ with common domain $\Omega$ to be a subset $E$ of $\Omega$ with the property that the restrictions to $E$ of the functions $f_{1}, \cdots, f_{m}$ are linearly independent. For example, every infinite subset of $\Omega$ with a limit point in $\Omega$ is a uniqueness set, as are "most" finite subsets of $\Omega$. In case $m=1$, a set $E \subset \Omega$ is a uniqueness set if and only if $f_{1}(z) \neq 0$ for some $z \in E$.

For notational convenience, we introduce the mapping $\lambda$ from numerical sequences $h$ to $d$-dimensional column vectors $H$ defined by 


$$
(\lambda h)_{k}=H_{k}=\left[\begin{array}{c}
h_{k d} \\
h_{k d+1} \\
\vdots \\
h_{k d+d-1}
\end{array}\right], k=0,1,2, \cdots
$$

We now state our principal results.

THEOREM 1. Suppose that (2.1)-(2.4) hold and let $h$ be a complex sequence. Then the following are equivalent:

(i) $\sum_{k=0}^{\infty} \alpha_{k}^{(\lambda h)_{k}}$ converges;

(ii) $h$ belongs to the domain of $B$;

(iii) $\sum_{k=0}^{\infty} h_{k} p_{k}(z)$ converges for all $z$ in some uniqueness set for $\varphi_{1}, \varphi_{2}, \cdots, \varphi_{d}$;

(iv) $\sum_{k=0}^{\infty} h_{k} p_{k}(z)$ is uniformly convergent on compact subsets of $\Omega$.

THEOREM 2. Suppose that (2.1)-(2.4) hold and let $h$ and $y$ be complex sequences. Then $y=B h$ if and only if $\sum_{k=0}^{\infty} h_{k} p_{k}(z)$ is uniformly convergent on compact subsets of $\Omega$ to the function $f$ whose power series at 0 is given by

$$
f(z)=\sum_{j=0}^{\infty} y_{j} z^{j} / j !
$$

The problem of explicitly determining those sequences $y$ which belong to the space $\mathscr{Y}$ is extremely difficult, even for very simple sequences $\left\{p_{k}\right\}_{0}^{\infty}$ (cf. [2]). The coefficient space $\mathscr{H}$ is, however, much more accessible, and conditions (2.1)-(2.4) allow us to characterize it completely.

For each integer $i, 1 \leqq i \leqq d$, let $S^{(i)}$ denote the complex sequence with terms given by

$$
S_{k d+j-1}^{(i)}=\left(\alpha_{k}\right)_{i j}, 1 \leqq j \leqq d, 0 \leqq k<\infty .
$$

THEOREM 3. Suppose that (2.1)-(2.4) hold. A complex sequence $h$ belongs to $\mathscr{H}$ if and only if each of the series

$$
\sum_{k=0}^{\infty} S_{k}^{(i)} h_{k}, 1 \leqq i \leqq d
$$

is convergent.

We note here for later use that if (2.5) converges, then

$$
\sum_{k=0}^{\infty} S_{k}^{(i)} h_{k}=\left(\sum_{k=0}^{\infty} \alpha_{k}(\lambda h)_{k}\right)_{i}, \quad 1 \leqq i \leqq d
$$


The expansions discussed in [2] and [3] share two interesting and apparently unrelated properties. The first of these is uniqueness: if $\sum_{k=0}^{\infty} h_{k} p_{k}(z)=0$ for all $z \in \Omega$, then $h_{k}=0, k=0,1,2, \cdots$. The second is the existence of an underlying Banach space structure; that is, one can define a norm $\|\cdot\|$ on $\mathscr{F}$, under which $\mathscr{F}$ is complete, such that convergence with respect to $\|\cdot\|$ implies uniform convergence on compact subsets of $\Omega$, and such that $\left\{p_{k}\right\}_{0}^{\infty}$ is a strong basis for the Banach space $\mathscr{F}$. In the present setting we show that the first of these properties implies the second, and also that $\mathscr{F}$ is isomorphic to $c_{0}$.

THEOREM 4. Suppose in addition to (2.1)-(2.4) that

$$
\begin{aligned}
\sum_{k=0}^{\infty} h_{k} p_{k}(z) & =0 \text { for all } z \in \Omega \text { implies } \\
\text { that } h_{k} & =0, k=0,1,2, \cdots .
\end{aligned}
$$

For each $f \in \mathscr{F}$ with

set

$$
f(z)=\sum_{k=0}^{\infty} h_{k} p_{k}(z)
$$

$$
\|f\|=\sup _{0 \leqq n<\infty}\left\|\sum_{k=n}^{\infty} \alpha_{k}(\lambda h)_{k}\right\|_{\tau} .
$$

Then $\mathscr{F}$ is a Banach space, $\left\{p_{k}\right\}_{0}^{\infty}$ is a basis for $\mathscr{F}$, and the mapping

$$
\Lambda f=\lambda^{-1}\left\{\sum_{k=n}^{\infty} \alpha_{k}(\lambda h)_{k}\right\}_{n=0}^{\infty}
$$

defines an isomorphism between $\mathscr{F}$ and $c_{0}$.

Note that the uniqueness assumption (2.7) is, in view of Theorem 2, equivalent to the assertion that the operator $B$ is one-to-one.

3. Proofs of theorems. The following lemma will be used in the proof of Theorem 1 .

Lemma 1. Suppose that $\left\{u_{k}\right\}_{0}^{\infty}$ is a sequence of $d \times d$ invertible matrices such that

$$
\sum_{k=0}^{\infty}\left\|u_{k+1}-u_{k}\right\|_{G}<\infty
$$

and that $\left\{u_{k}\right\}_{0}^{\infty}$ converges elementwise to an invertible matrix. Then if 
$\left\{v_{k}\right\}_{0}^{\infty}$ is a sequence of column vectors, $\sum_{k=0}^{\infty} v_{k}$ converges if and only if $\sum_{k=0}^{\infty} u_{k} v_{k}$ converges.

Proof. Suppose $\Sigma_{0}^{\infty} v_{k}$ converges. Writing $s_{n}=v_{o}+\cdots+v_{n}$, we have $\sum_{k=0}^{n} u_{k} v_{k}=u_{n} s_{n}+\sum_{k=0}^{n-1}\left(u_{k}-u_{k+1}\right) s_{k}$. Since $\sum_{k=0}^{\infty}\left\|\left(u_{k}-u_{k+1}\right) s_{k}\right\|_{\tau} \leqq$ $d \sum_{k=0}^{\infty}\left\|u_{k}-u_{k+1}\right\| G\left\|s_{k}\right\|_{\tau}<\infty$, the convergence of $\Sigma_{0}^{\infty} u_{k} v_{k}$ follows.

Now suppose that $\Sigma_{0}^{\infty} u_{k} v_{k}$ converges, set $\sigma_{n}=u_{0} v_{0}+\cdots+u_{n} v_{n}$, and note that $\sum_{k=0}^{n} v_{k}=\sum_{k=0}^{n} u_{k}^{-1}\left(u_{k} v_{k}\right)=u_{n}^{-1} \sigma_{n}+\sum_{k=0}^{n-1} u_{k}^{-1}\left(u_{k+1}-u_{k}\right) u_{k+1}^{-1} \sigma_{k}$. Since $\left\{\boldsymbol{u}_{k}\right\}_{0}^{\infty}$ converges to an invertible matrix, say $u$, then $\left\{\boldsymbol{u}_{k}^{-1}\right\}_{0}^{\infty}$ must converge to $u^{-1}$. Consequently, the sequence $\left\{u_{n}^{-1} \sigma_{n}\right\}_{0}^{\infty}$ converges and the sequence $\left\{\left\|u_{k}^{-1}\right\|_{G}\right\}_{0}^{\infty}$ is bounded. In view of

$$
\left\|u_{k}^{-1}\left(u_{k+1}-u_{k}\right) u_{k+1}^{-1} \sigma_{k}\right\|_{\tau} \leqq d^{3}\left\|u_{k+1}-u_{k}\right\|_{G}\left\|u_{k+1}^{-1}\right\|_{G}\left\|u_{k+1}^{-1}\right\|_{G}\left\|\sigma_{k}\right\|_{r}
$$

(3.1) shows that $\Sigma_{0}^{\infty} v_{k}$ converges, and this completes the proof.

We will require the following lemma on linearly independent sets of functions. The proof is omitted.

Lemma 2. Let $E$ be a uniqueness set for $f_{1}, f_{2}, \cdots, f_{m}$. Then there are points $z_{1}, z_{2}, \cdots, z_{m}$ in $E$ such that the matrix $\left(f_{j}\left(z_{k}\right)\right), 1 \leqq j, k \leqq d$, is invertible. If $f_{1}, \cdots, f_{m}$ are analytic in $\Omega$, then there exist nonnegative integers $n_{1}, n_{2}, \cdots, n_{m}$ such that the matrix $\left(f_{j}^{\left(n_{k}\right)}(0)\right), 1 \leqq j, k \leqq d$, is invertible.

Lemma 3. Suppose that (2.1)-(2.3) hold and let $h$ be a complex sequence such that $\sum_{k=0}^{\infty} \alpha_{k}(\lambda h)_{k}$ converges. Then

$$
\sum_{k=0}^{\infty} h_{k} p_{k}(z)
$$

converges if and only if

$$
\sum_{k=0}^{\infty} P_{k}(z)(\lambda h)_{k}
$$

converges. Moreover, (3.2) converges uniformly on compact subsets of $\Omega$ if and only if the same is true of (3.3).

Proof. Since

$$
\sum_{k=0}^{\infty} P_{k}(z)(\lambda h)_{k}=\sum_{k=0}^{\infty} \sum_{t=0}^{d-1} h_{k d+t} p_{k d+t}(z),
$$

then clearly convergence of (3.2) implies convergence of (3.3).

In the other direction, it is sufficient to prove that, for fixed $t$, 


$$
\lim _{n \rightarrow \infty} h_{n d+t} p_{n d+t}(z)=0
$$

uniformly on compact subsets of $\Omega$. Let $\left\{x_{k}\right\}_{0}^{\infty}$ be the sequence of column vectors defined by

$$
x_{k}=\sum_{j=k}^{\infty} \alpha_{j}(\lambda h)_{j}, \quad k=0,1,2, \cdots
$$

and define

$$
Q_{k}(z)=P_{k}(z) \alpha_{k}^{-1}-P_{k-1}(z) \alpha_{k-1}^{-1}, \quad k=0,1,2, \cdots
$$

Then $(\lambda h)_{k}=\alpha_{k}^{-1}\left(x_{k}-x_{k+1}\right)$ and $P_{k}(z)=\sum_{j=0}^{k} Q_{j}(z) \alpha_{k}, 0 \leqq k<\infty$. Therefore

$$
\begin{aligned}
\left|h_{n d+t} p_{n d+t}(z)\right| & =\left|\left((\lambda h)_{n}\right)_{t}\left(P_{n}(z)\right)_{t}\right| \\
& =\left|\left(\alpha_{n}^{-1}\left(x_{n}-x_{n+1}\right)\right)_{t}\right|\left|\left(\sum_{j=0}^{n} Q_{j}(z) \alpha_{n}\right)\right|_{t} \\
& \leqq\left\|\alpha_{n}^{-1}\left(x_{n}-x_{n+1}\right)\right\|_{\tau}\left\|\sum_{j=0}^{n} Q_{j}(z) \alpha_{n}\right\|_{\rho} \\
& \leqq d^{2}\left\|\alpha_{n}^{-1}\right\|_{G}\left\|x_{n}-x_{n+1}\right\|\left\|\alpha_{n}\right\|_{G}\left\|\sum_{j=0}^{n} Q_{j}(z)\right\|_{\rho}
\end{aligned}
$$

Since $\left\{\left(x_{n}-x_{n+1}\right)\right\}_{0}^{\infty}$ converges to the zero vector, the result follows from (2.3). The uniform convergence is a consequence of (2.2).

In view of Lemma 3, Theorem 1 is equivalent to the following result.

Theorem 5. Suppose that (2.1)-(2.4) hold and let $h$ be a complex sequence. Then the following are equivalent:

(i) $\sum_{k=0}^{\infty} \alpha_{k}(\lambda h)_{k}$ converges ;

(ii) $\quad \sum_{k=0}^{\infty} P_{k}^{(j)}(0)(\lambda h)_{k}$ converges for $j=0,1,2, \cdots$,

(iii) $\sum_{k=0}^{\infty} P_{k}(z)(\lambda h)_{k}$ converges for all $z$ in some uniqueness set for $\varphi_{1}, \varphi_{2}, \cdots, \varphi_{d}$;

(iv) $\sum_{k=0}^{\infty} P_{k}(z)(\lambda h)_{k}$ converges uniformly on compact subsets of $\Omega$.

Proof. We first show that (i) and (iii) are equivalent. Using Lemma 2 and (2.4), choose points $z_{1}, z_{2}, \cdots, z_{d}$ in $\Omega$ such that the matrix $\left(\varphi_{j}\left(z_{k}\right)\right), 1 \leqq j, k \leqq d$, is invertible. In proving that (i) implies (iii) we can take $\left\{z_{1}, \cdots, z_{d}\right\}$ as our uniqueness set; for the reverse implication we will suppose that $z_{1}, \cdots, z_{d}$ are chosen from some arbitrary uniqueness set. 
Define

$$
\begin{aligned}
u_{k} & =\left[\begin{array}{c}
P_{k}\left(z_{1}\right) \\
\vdots \\
P_{k}\left(z_{d}\right)
\end{array}\right]_{\alpha_{k}^{-1},} \quad k=0,1,2, \cdots \\
u & =\left[\begin{array}{c}
\Phi\left(z_{1}\right) \\
\vdots \\
\Phi\left(z_{d}\right)
\end{array}\right]
\end{aligned}
$$

and let $v_{k}=\alpha_{k}(\lambda h)_{k}$. Then $u$ is invertible, $\left\{u_{k}\right\}_{0}^{\infty}$ converges to $u$, and (iii) holds if and only if $\Sigma_{0}^{\infty} u_{k} v_{k}$ converges. Note also that $u_{k}$ is invertible for $k$ sufficiently large, and we suppose without loss of generality that this is true for all $k$. By Lemma 1, then, we can prove the equivalence of (i) and (iii) by showing that

$$
\sum_{k=0}^{\infty}\left\|u_{k+1}-u_{k}\right\|_{G}<\infty
$$

For $0 \leqq k<\infty$, we have

$$
\begin{aligned}
\left\|u_{k+1}-u_{k}\right\|_{G} & =\sum_{i=1}^{d} \sum_{t=1}^{d}\left|\left(P_{k+1}\left(z_{t}\right) \alpha_{k+1}^{-1}-P_{k}\left(z_{t}\right) \alpha_{k}^{-1}\right)_{i}\right| \\
& \leqq d \sum_{t=1}^{d}\left\|P_{k+1}\left(z_{t}\right) \alpha_{k+1}^{-1}-P_{k}\left(z_{t}\right) \alpha_{k}^{-1}\right\|_{\rho}
\end{aligned}
$$

and thus

$$
\sum_{k=0}^{\infty}\left\|u_{k+1}-u_{k}\right\|_{G} \leqq d \sum_{t=1}^{d} \sum_{k=1}^{\infty}\left\|P_{k}\left(z_{t}\right) \alpha_{k}^{-1}-P_{k-1}\left(z_{t}\right) \alpha_{k-1}^{-1}\right\|_{\rho}<\infty .
$$

We now prove that (i) $\Rightarrow$ (iv) $\Rightarrow$ (ii) $\Rightarrow$ (i). Thus suppose that $\Sigma_{0}^{\infty} \alpha_{k}(\lambda h)_{k}$ converges and define $Q_{k}(z), 0 \leqq k<\infty$, as in (3.5). Then

$$
\begin{gathered}
\sum_{k=0}^{n} P_{k}(z)(\lambda h)_{k}=\sum_{k=0}^{n}\left\{\sum_{j=0}^{k} Q_{j}(z) \alpha_{k}\right\}(\lambda h)_{k} \\
=\sum_{j=0}^{n} \sum_{k=j}^{n} Q_{j}(z) \alpha_{k}(\lambda h)_{k}=\sum_{j=0}^{n} Q_{j}(z)\left\{\sum_{k=j}^{\infty} \alpha_{k}(\lambda h)_{k}-\sum_{k=n+1}^{\infty} \alpha_{k}(\lambda h)_{k}\right\} \\
=\sum_{j=0}^{n} Q_{j}(z) \sum_{k=j}^{\infty} \alpha_{k}(\lambda h)_{k}-\sum_{j=0}^{n} Q_{j}(z) \sum_{k=n+1}^{\infty} \alpha_{k}(\lambda h)_{k} \\
=\sum_{i=0}^{n} Q_{j}(z) \sum_{k=j}^{\infty} \alpha_{k}(\lambda h)_{k}-\left(P_{n}(z) \alpha_{n}^{-1}\right) \sum_{k=n+1}^{\infty} \alpha_{k}(\lambda h)_{k} .
\end{gathered}
$$


Let $\epsilon>0$ and choose an integer $N>0$ such that $j \geqq N$ implies $\left\|\sum_{k=j}^{\infty} \alpha_{k}(\lambda h)_{k}\right\|_{\tau}<\epsilon$. Then $n \geqq N$ implies

$$
\begin{gathered}
\sum_{j=0}^{n}\left|Q_{j}(z)\left(\sum_{k=j}^{\infty} \alpha_{k}(\lambda h)_{k}\right)\right| \\
\leqq \sum_{j=0}^{N-1}\left|Q_{j}(z)\left(\sum_{k=j}^{\infty} \alpha_{k}(\lambda h)_{k}\right)\right|+d \epsilon \sum_{j=N}^{n}\left\|Q_{j}(z)\right\|_{\rho} .
\end{gathered}
$$

Since $P_{n}(z) \alpha_{n}^{-1}$ converges to $\Phi(z)$, the last term in (3.6) converges to 0 . Combining this with (3.7) we see that $\sum_{k=0}^{\infty} P_{k}(z)(\lambda h)_{k}$ converges uniformly on compact subsets of $\Omega$. This proves that (i) $\Rightarrow$ (iv).

The implication (iv) $\Rightarrow$ (ii) is obvious, so there remains only to show that (ii) $\Rightarrow$ (i). Choose, via Lemma 2 , integers $j_{1}, j_{2}, \cdots, j_{d}$ such that the matrix

$$
u=\left[\begin{array}{c}
\Phi^{\left.()_{1}\right)}(0) \\
\vdots \\
\Phi^{\left(j_{d}\right)}(0)
\end{array}\right]
$$

is invertible. Let

$$
u_{k}=\left[\begin{array}{c}
P_{k}^{\left(j_{1}\right)}(0) \\
\vdots \\
P_{k}^{\left(j_{\alpha}\right)}(0)
\end{array}\right] \alpha_{k}^{-1}, v_{k}=\alpha_{k}(\lambda h)_{k}, \quad k=0,1,2, \cdots,
$$

and note that (ii) implies that $\sum_{0}^{\infty} u_{k} v_{k}$ converges. Moreover, $\left\{u_{k}\right\}_{0}^{\infty}$ converges to $u, u_{k}$ is invertible for $k$ sufficiently large, and we may again assume that $u_{k}$ is invertible for all $k$. To show that $\Sigma_{0}^{\infty} v_{k}$ converges, we use Lemma 1. First, the convergence of

$$
\sum_{k=0}^{\infty}\left\|P_{k}^{(j)}(0) \alpha_{k}^{-1}-P_{k-1}^{(j)}(0) \alpha_{k-1}^{-1}\right\|_{\rho}, \quad 0 \leqq j<\infty
$$

follows easily from (2.2). Now observe that

$$
\begin{aligned}
\left\|u_{k+1}-u_{k}\right\|_{G} & =\sum_{i=1}^{d} \sum_{t=1}^{d}\left|\left(P_{k+1}^{\left(j_{1}\right)}(0) \alpha_{k+1}^{-1}-P_{k}^{\left(j_{i}\right)}(0) \alpha_{k}^{-1}\right)_{t}\right| \\
& \leqq d \sum_{i=1}^{d}\left\|P_{k+1}^{\left(j_{i}\right)}(0) \alpha_{k+1}^{-1}-P_{k}^{\left(j_{i}\right)}(0) \alpha_{k}^{-1}\right\|_{\rho}
\end{aligned}
$$

so that 


$$
\sum_{k=0}^{\infty}\left\|u_{k+1}-u_{k}\right\|_{G} \leqq d \sum_{i=1}^{d} \sum_{k=0}^{\infty}\left\|P_{k+1}^{\left(j_{j}\right)}(0) \alpha_{k+1}^{-1}-P_{k}^{\left(i_{1}\right)}(0) \alpha_{k}^{-1}\right\|_{\rho}<\infty
$$

and this completes the proof of the theorem.

Proof of Theorem 2. Suppose $y=B h$ for complex sequences $y$ and $h$. Since $h$ belongs to the domain of $B$, Theorem 1 implies that $\sum_{k=0}^{\infty} h_{k} p_{k}(z)$ converges uniformly on compact subsets of $\Omega$. Let $f(z)=$ $\sum_{k=0}^{\infty} h_{k} p_{k}(z)$. Then

$$
f^{(j)}(0)=\sum_{k=0}^{\infty} h_{k} p_{k}^{(j)}(0)=\sum_{k=0}^{\infty} B_{j k} h_{k}=(B h)_{j}=y_{j}
$$

$0 \leqq j<\infty$.

In the other direction, if

$$
\sum_{j=0}^{\infty} y_{j} \frac{z^{j}}{j !}=\sum_{k=0}^{\infty} h_{k} p_{k}(z)
$$

then differentiating this equation $m$ times and setting $z=0$ yields

$$
y_{m}=\sum_{k=0}^{\infty} h_{k} p_{k}^{(m)}(0)=(B h)_{m},
$$

so that $y=B h$.

Proof of Theorem 3. If $h \in \mathscr{H}$, then $h$ belongs to the domain of B. By Theorem 1, $\sum_{k=0}^{\infty} \alpha_{k}(\lambda h)_{k}$ converges. By definition of the sequences $\left\{S_{k}^{(i)}\right\}_{k=0}^{\infty}, 1 \leqq i \leqq d$, it follows that each of the series

$$
\sum_{k=0}^{\infty} \sum_{j=1}^{d} S_{k d+j-1}^{(i)} h_{k d+d-1}, \quad 1 \leqq i \leqq d
$$

is convergent. To show that the series (2.5) all converge, it suffices to show that, for fixed $j, 1 \leqq j \leqq d$, and fixed $i$, the terms $S_{k d+\jmath-1}^{(i)} h_{k d+\jmath-1} \rightarrow 0$ as $k \rightarrow \infty$. As in the proof of Lemma 3, let $x_{k}=\sum_{j=k}^{\infty} \alpha_{j}(\lambda h)_{j}, k=$ $0,1,2, \cdots$. Then $(\lambda h)_{k}=\alpha_{k}^{-1}\left(x_{k}-x_{k+1}\right), 0 \leqq k<\infty$, and

$$
\begin{aligned}
\left|S_{k d+j-1}^{(i)} h_{k d+j-1}\right| & =\left|\left(\alpha_{k}\right)_{i j}\left((\lambda h)_{k}\right)_{j}\right| \\
& \leqq\left|\left(\alpha_{k}\right)_{i j}\right|\left\|(\lambda h)_{k}\right\|_{\tau}=\left|\left(\alpha_{k}\right)_{i j}\right|\left\|\alpha_{k}^{-1}\left(x_{k}-x_{k+1}\right)\right\|_{\tau} \\
& \leqq d\left\|\alpha_{k}\right\|_{G}\left\|\alpha_{k}^{-1}\right\|_{G}\left\|x_{k}-x_{k+1}\right\|_{\tau} .
\end{aligned}
$$


Since $\left\{x_{k}-x_{k+1}\right\}_{0}^{\infty}$ converges to the zero vector, the desired result follows.

If each of the series (2.5) converges, then each of the "grouped series"

$$
\sum_{k=0}^{\infty} \sum_{j=1}^{d} S_{k d+j-1}^{(i)} h_{k d+j-1}
$$

converges. Therefore $\Sigma_{0}^{\infty} \alpha_{k}(\lambda h)_{k}$ converges and Theorem 1 implies $h \in \mathscr{H}$.

Proof of Theorem 4. Let $n$ be a nonnegative integer and write $n=m d+i, 0 \leqq i<d$. Then

$$
(\Lambda f)_{n}=\sum_{k=m d}^{\infty} S_{k}^{(i+1)} h_{k}
$$

If $\|\cdot\|_{\infty}$ denotes the supremum norm on $c_{0}$, then

$$
\begin{aligned}
\|\Lambda f\|_{\infty} & =\sup _{0 \leqq m<\infty}\left\{\max _{0 \leqq i<d}\left|\sum_{k=m d}^{\infty} S_{k}^{(i+1)} h_{k}\right|\right\} \\
& =\sup _{0 \leqq m<\infty}\left\|\sum_{k=m}^{\infty} \alpha_{k}(\lambda h)_{k}\right\|_{\tau} \\
& =\|f\| .
\end{aligned}
$$

Thus $\Lambda$ is an isometry. Since $\Lambda$ maps $\mathscr{F}$ onto all of $c_{0}$, it follows that $\mathscr{F}$ is a Banach space.

Let $f \in \mathscr{F}$, with

$$
f(z)=\sum_{k=0}^{\infty} h_{k} p_{k}(z)
$$

For each positive integer $n$, write $n=j d+i, j \geqq 0,0 \leqq i<d$. Then

$$
\begin{aligned}
\left\|f(z)-\sum_{k=0}^{n} h_{k} p_{k}(z)\right\| & =\left\|\sum_{k=n+1}^{\infty} h_{k} p_{k}(z)\right\| \\
& =\sup _{m \geqq j}\left\|\sum_{k=m}^{\infty} \alpha_{k}(\lambda h)_{k}\right\|_{\tau},
\end{aligned}
$$

and this last expression tends to 0 as $n \rightarrow \infty$. Thus (3.7) converges in the norm $\|\cdot\|$. From the uniqueness condition (2.7), it follows that $\left\{p_{k}\right\}_{0}^{\infty}$ is a basis for $\mathscr{F}$. 


\section{REFERENCES}

1. R. P. Boas and R. C. Buck, Polynomial Expansions of Analytic Functions, Springer, Berlin, 1964.

2. J. D. Buckholtz, Series expansions of.analytic functions, J. Math. Anal. and Appl., 41 (3) (1973), 673-684.

3. - Appell polynomial expansions and biorthogonal expansions in Banach spaces, Trans. Amer. Math. Soc., 181 (1973), 245-272.

4. J. D. Buckholtz and J. K. Shaw, On functions expandable in Lidstone series J. Math. Anal. and Appl., 47 (3) (1974), 626-632.

5. W. T. Martin, On expansions in terms of a general class of functions, Amer. J. Math.. 58 (1936), $407-420$.

Received December 11, 1973.

UNIVERSITY OF KENTUCKY

AND

Virginia Polytechnic Institute and State University 


\section{PACIFIC JOURNAL OF MATHEMATICS \\ EDITORS}

\author{
Richard ARENS (Managing Editor) \\ University of California \\ Los Angeles, California 90024
}

\author{
R. A. Beaumont \\ University of Washington \\ Seattle, Washington 98105
}

\section{J. DugundJI}

Department of Mathematics

University of Southern California

Los Angeles, California 90007

D. Gilbarg and J. Milgram

Stanford University

Stanford, California 94305

\section{ASSOCIATE EDITORS}
E. F. BECKENBACH
B. H. NeumanN
F. WOLF
K. YoshidA

\section{SUPPORTING INSTITUTIONS}

\author{
UNIVERSITY OF BRITISH COLUMBIA \\ CALIFORNIA INSTITUTE OF TECHNOLOGY \\ UNIVERSITY OF CALIFORNIA \\ MONTANA STATE UNIVERSITY \\ UNIVERSITY OF NEVADA \\ NEW MEXICO STATE UNIVERSITY \\ OREGON STATE UNIVERSITY \\ UNIVERSITY OF OREGON \\ OSAKA UNIVERSITY
}

\author{
UNIVERSITY OF SOUTHERN CALIFORNIA \\ STANFORD UNIVERSITY \\ UNIVERSITY OF TOKYO \\ UNIVERSITY OF UTAH \\ WASHINGTON STATE UNIVERSITY \\ UNIVERSITY OF WASHINGTON \\ AMERICAN MATHEMATICAL SOCIETY
}

The Supporting Institutions listed above contribute to the cost of publication of this Journal, but they are not owners or publishers and have no responsibility for its contents or policies.

Mathematical papers intended for publication in the Pacific Journal of Mathematics should be in typed form or offset-reproduced (not dittoed), double spaced with large margins. Underline Greek letters in red, German in green, and script in blue. The first paragraph or two must be capable of being used separately as a synopsis of the entire paper. Items of the bibliography should not be cited there unless absolutely necessary, in which case they must be identified by author and Journal, rather than by item number. Manuscripts, in duplicate, may be sent to any one of the four editors. Please classify according to the scheme of Math. Reviews, Index to Vol. 39. All other communications should be addressed to the managing editor, or Elaine Barth, University of California, Los Angeles, California, 90024.

100 reprints are provided free for each article, only if page charges have been substantially paid. Additional copies may be obtained at cost in multiples of 50 .

The Pacific Journal of Mathematics is issued monthly as of January 1966. Regular subscription rate: $\$ 72.00$ a year (6 Vols., 12 issues). Special rate: $\$ 36.00$ a year to individual members of supporting institutions.

Subscriptions, orders for back numbers, and changes of address should be sent to Pacific Journal of Mathematics, 103 Highland Boulevard, Berkeley, California, 94708.

PUBLISHED BY PACIFIC JOURNAL OF MATHEMATICS, A NON-PROFIT CORPORATION Printed at Jerusalem Academic Press, POB 2390, Jerusalem, Israel.

$$
\begin{gathered}
\text { Copyright (c) } 1975 \text { Pacific Journal of Mathematics } \\
\text { All Rights Reserved }
\end{gathered}
$$




\section{Pacific Journal of Mathematics}

Vol. 56, No. 2 December, 1975

Ralph Alexander, Generalized sums of distances .................. 297

Zvi Arad and George Isaac Glauberman, A characteristic subgroup of a group of odd order ............................... 305

B. Aupetit, Continuité du spectre dans les algèbres de Banach avec involution ........................................ 321

Roger W. Barnard and John Lawson Lewis, Coefficient bounds for some classes of starlike functions . ...........................

Roger W. Barnard and John Lawson Lewis, Subordination theorems for some classes of starlike fumctions . .................... 333

Ladislav Bican, Preradicals and injectivity .................. 367

James Donnell Buckholtz and Ken Shaw, Series expansions of analytic

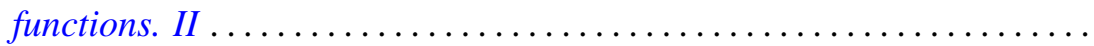

Richard D. Carmichael and E. O. Milton, Distributional boundary values in

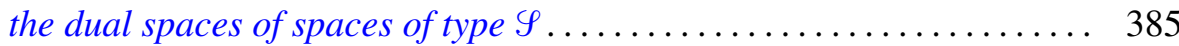

Edwin Duda, Weak-unicoherence ............................ 423

Albert Edrei, The Padé table of functions having a finite number of essential singularities ........................................

Joel N. Franklin and Solomon Wolf Golomb, A function-theoretic approach to the study of nonlinear recurring sequences ................ 455

George Isaac Glauberman, On Burnside's other $p^{a} q^{b}$ theorem 469

Arthur D. Grainger, Invariant subspaces of compact operators on topological vector spaces .........................

Jon Craig Helton, Mutual existence of sum and product integrals .

Franklin Takashi Iha, On boundary functionals and operators with finite-dimensional null spaces

Gerald J. Janusz, Generators for the Schur group of local and global number fields

A. Katsaras and Dar-Biau Liu, Integral representations of weakly compact operators.

W. J. Kim, On the first and the second conjugate points .

Charles Philip Lanski, Regularity and quotients in rings with involution ....

Ewing L. Lusk, An obstruction to extending isotopies of piecewise linear manifolds.

Saburou Saitoh, On some completenesses of the Bergman kernel and the Rudin kernel..

Stephen Jeffrey Willson, The converse to the Smith theorem for $Z_{p}$-homology spheres. 